\title{
METODOLOGÍAS Y EVALUACIÓN EN LOS TÍTULOS DE GRADO: ESTUDIO DE CASO DE LA MATERIA TEORÍA Y PRÁCTICA DE LAS RELACIONES PÚBLICAS
}

\author{
María Isabel Míguez-González¹: Universidad de Vigo. España \\ mabelm@uvigo.es
}

\section{RESUMEN}

Este estudio de caso describe las metodologías docentes y procedimientos de evaluación de la materia Teoría y práctica de las relaciones públicas, del título de grado en Publicidad y Relaciones Públicas de la Universidad de Vigo, con la finalidad de proponer mecanismos de mejora. Para ello se realiza un análisis y valoración de las dos ediciones en las que se ha impartido la asignatura, basado en la observación participante de la profesora, el análisis de contenido de las guías docentes, los resultados académicos obtenidos y las encuestas de evaluación docente respondidas por los alumnos. Según el análisis, la estructura de la materia permite la conjunción de diversas metodologías que, unidas a un sistema de evaluación diversificado, han llevado a la obtención de resultados muy positivos y a un elevado nivel de satisfacción de los alumnos, aunque a costa de una alta dedicación por parte del docente. Por ello, se proponen modificaciones en los ejercicios y el sistema de evaluación que faciliten el trabajo del docente sin perjudicar las posibilidades de adquisición de competencias por parte de los estudiantes.

PALABRAS CLAVE: Relaciones públicas - Docencia - EEES - Metodologías docentes - Evaluación

\footnotetext{
${ }^{1}$ Autor correspondiente

María Isabel Míguez-González1: Facultad de Ciencias Sociales y de la Comunicación. Universidad de Vigo. Pontevedra (España).

Correo: mabelm@uvigo.es
} 


\title{
CASE STUDY OF THE SUBJECT THEORY AND PRACTICE OF PUBLIC RELATIONS
}

\begin{abstract}
This case study describes the teaching methodologies and evaluation procedures of the subject Theory and practice of public relations, in the degree of Advertising and public relations of the University of Vigo, whit the aim of proposing ways to improve it. With this objective we have developed an analysis and evaluation of the two editions of the subject, based on direct observation, content analysis of teaching guides, academic results and teacher's evaluations surveys answered by the students. The analysis shows that the structure of the subject allows the combination of different methodologies. These procedures, joined to a diversified evaluation system, have led to positive academic outcomes and to a high level of student satisfaction, although at the expense of a high dedication by the teacher. Thus, we propose modifications of the exercises and the evaluation system to facilitate the teacher's work without harming the chances of acquiring skills by students.
\end{abstract}

KEY WORDS: Public Relations - Teaching - European Space for Higher Education - Teaching methodologies - Evaluation

\section{INTRODUCCIÓN}

La aplicación de las premisas establecidas en la Declaración de Bolonia (VV.AA.,1999) para el Espacio Europeo de la Educación Superior, matizadas y actualizadas en la Declaración de Lovaina (VV.AA., 2009), ha llevado a la conversión de las licenciaturas y diplomaturas impartidas en España en nuevos títulos de grado. Esto no sólo ha significado una reestructuración de objetivos, materias y fórmulas de organización de la docencia, sino que ha implicado una importante revisión de las metodologías y mecanismos de evaluación que se aplican en las titulaciones.

Así, de una orientación basada en la figura del docente, en sus habilidades a la hora de impartir la lección magistral y en la evaluación de resultados a partir de la demostración de conocimientos en una prueba oral o escrita, se ha pasado o se debería pasar a un enfoque didáctico centrado en la figura del alumno, en el desarrollo de sus competencias y con fórmulas diversas para medir la adquisición de dichas competencias, más allá de la mera comprobación de conocimientos. 
En este contexto, tal y como apunta Palomares Ruiz, "la práctica profesional del docente debe convertirse en un proceso de acción y reflexión cooperativa, de indagación y experimentación, donde el profesor aprende a enseñar y enseña porque aprende, interviene para facilitar -y no imponer ni sustituir- la comprensión del alumnado $\mathrm{y}$, al reflexionar sobre su intervención, ejerce y desarrolla su propio entendimiento." (Palomares Ruiz, 2001, p. 593).

Esta nueva perspectiva se ha ido incorporando, con las modificaciones que implica, a los planes de estudio de las nuevas titulaciones. Pero todos los cambios que se van introduciendo, precisamente por su carácter experimental, han de ser revisados y valorados para comprobar su eficacia. En este sentido, son numerosos los congresos y publicaciones que recogen las experiencias de docentes de diversos ámbitos y que están contribuyendo a clarificar la utilidad y posibilidades de distintas metodologías y procedimientos de evaluación en el marco real de las universidades españolas, con los condicionantes de tiempo, recursos o número de alumnos con los que el profesor se encuentra a la hora de renovar una materia de acuerdo con el espíritu del EEES.

En esta línea se enmarca el presente estudio, que plantea una revisión de una materia de grado del ámbito de la comunicación.

\section{METODOLOGÍA}

\subsection{Objetivos del estudio}

El objetivo de esta investigación consiste en realizar una aproximación descriptiva al programa, metodologías docentes y procedimientos de evaluación de la materia Teoría y práctica de las relaciones públicas, impartida en el $1^{\mathrm{er}}$ curso del título de grado de Publicidad y Relaciones Públicas de la Universidad de Vigo, con la finalidad de proponer posibles mecanismos de mejora.

\subsection{Metodología}

El objetivo propuesto implica el análisis y valoración de las dos primeras ediciones en las que se ha impartido la asignatura (curso 2009-2010 y curso 2010-2011). Este análisis se realiza tanto desde el punto de vista de los resultados académicos y del nivel de cumplimiento de competencias como desde la óptica de la propia experiencia de ejecución y desarrollo de la docencia.

Para ello se ha desarrollado un proceso de observación participante por parte de la docente de la materia. Los resultados de esta observación se complementan con el análisis de contenido de las guías docentes (fundamentadas en la memoria de titulación aprobada por la ANECA), de los resultados académicos obtenidos y de las encuestas de evaluación docente respondidas por los alumnos. 


\subsection{Descripción básica de la materia}

\section{Ubicación en el plan de estudios}

El título de grado en Publicidad y Relaciones Públicas de a Universidad de Vigo se organiza en tres módulos: un módulo transversal (con materias básicas que el alumno debe conocer para contar con una base adecuada en ciencias sociales y en comunicación en general); un segundo módulo dedicado a la comunicación publicitaria y un tercer módulo orientado a las relaciones públicas, la comunicación corporativa y la comunicación institucional.

La materia Teoría y práctica de las relaciones públicas, que se imparte en el 1 er curso, es la primera materia del módulo de relaciones públicas que cursan los alumnos y debe servir como base para el desarrollo de materias posteriores como Imagen corporativa ( $2^{\circ}$ curso), Planificación estratégica de relaciones públicas (3er curso) y Técnicas de relaciones públicas (3er curso). Además, también en $3^{\circ}$, la docencia del módulo se refuerza con una orientación al ámbito institucional y político mediante las materias Técnicas de propaganda política y Técnicas de comunicación electoral e institucional.

El módulo se completa en el $4^{\circ}$ curso con una materia obligatoria orientada a la comunicación corporativa, Dirección de comunicación, y con la oferta de cuatro materias optativas que cubren aspectos específicos de la disciplina: Relaciones públicas y protocolo, Comunicación de crisis, Relaciones públicas y comunicación solidaria y Campañas de relaciones públicas. El alumno puede cursar todas las materias optativas de este módulo o puede combinarlas con las optativas de otros módulos.

Con este mapa de materias se pretende cubrir las necesidades formativas básicas del alumno en este ámbito atendiendo a las recomendaciones y sugerencias de diferentes foros, como las de la Comisión sobre Educación de Relaciones Públicas estadounidense resumidas por Wilcox, Cameron y Xifra (2006, p. 29-30) o, ya en España, las del II Foro Interuniversitario de profesores de Relaciones Públicas, celebrado en VIC en 2004.

\section{Estructura}

Tanto la materia que nos ocupa como la mayoría de las restantes materias del grado han sido dotadas de una estructura similar en la que se reduce la presencialidad del alumno en las sesiones teóricas en relación con la antigua licenciatura y se prima el componente práctico y de trabajo en grupos pequeños.

Así, las materias cuentan con 6 créditos ECTS que implican, para el estudiante, 25 horas de docencia teórica presencial en grupos de 50-60 alumnos, más un total de 25 horas de docencia en grupos reducidos (10 horas de seminarios y 15 horas de 
en grupos numerosos. El resto de horas se corresponden con el trabajo que el alumno debe realizar fuera del aula.

En la primera edición, las 15 horas de práctica se desarrollaron, al igual que la teoría, en dos grupos de 50-60 alumnos, mientras que las horas de seminario se impartieron en cinco grupos de 20-25 alumnos. Teniendo en cuenta que la amplitud de los grupos de práctica dificultaba la aplicación de algunas metodologías, en la segunda edición (tanto de esta como de otras materias) se modificó la estructura y tanto los seminarios como las prácticas pasaron a impartirse en cuatro grupos de 25-30 alumnos. Esto no sólo facilitó la aplicación de metodologías difíciles de implantar en grupos amplios, sino que también permitió al profesor flexibilizar la docencia impartiendo seminarios o prácticas en las horas dedicadas a tal efecto según las necesidades de la materia.

\section{Competencias}

Tal y como destaca el profesor De Miguel (2006) la planificación didáctica en el Espacio Europeo de la Educación Superior ha de estar centrada, más que en los conocimientos, en las competencias que deben adquirir los estudiantes de una determinada titulación o asignatura.

La materia Teoría y práctica de las relaciones públicas, al ser la primera en la que se abordan contenidos relativos a la disciplina dentro del plan de estudios, cuenta con un importante componente de carácter teórico, relativo al análisis conceptual de diferentes elementos y a la comprensión de diversos modelos formulados por los especialistas en relaciones públicas. Pero, teniendo en cuenta la orientación y los preceptos de Bolonia, se ha intentado completar este componente teórico con contenidos y metas de tipo práctico encaminadas, desde un punto de vista preliminar, a favorecer la realización de un trabajo de calidad cuando el alumno se incorpore al ámbito profesional. Teniendo esto en cuenta, al cursar esta materia el alumno debería adquirir las siguientes competencias: 


\section{Saber}

1. Conocer el concepto de relaciones públicas, sus implicaciones, la historia de la disciplina y su evolución.

2. Analizar y comprender las diferentes corrientes y perspectivas de estudio de las relaciones

públicas.

3. Identificar y describir las funciones de las relaciones públicas en el contexto empresarial e institucional.

4. Identificar y analizar los sujetos que intervienen en la actividad de las relaciones públicas.

5. Reconocer los roles profesionales de la disciplina y profundizar en sus particularidades

6. Conocer las distintas fases del proceso de relaciones públicas

7. Conocer y comprender el marco ético y legal por el que se rige la práctica profesional de la disciplina

8. Asumir la importancia de las relaciones públicas en el contexto económico, político, sociocultural, tecnológico y comunicativo

\section{Ser/saber estar}

9. Contribuir al análisis activo de los elementos de las relaciones públicas, con la finalidad de generar conocimientos en esta disciplina

10. Desarrollar la capacidad de análisis, síntesis y juicio crítico

11. Tomar conciencia de la adecuada gestión del tiempo y organización de tareas

12. Esforzarse en gestionar de forma eficaz el tiempo en las tareas individuales y grupales

13. Aceptar y asumir los referentes éticos de la disciplina

14. Comprometerse a actuar con libertad y responsabilidad en el ejercicio de la disciplina a partir de dichos referentes éticos

15. Cuidar las relaciones con otras personas y con el entorno a partir de la propia identidad y valores

16. Colaborar en el trabajo en equipo a través del uso de la comunicación oral y escrita 
Tabla 2. Programa de contenidos y competencias asociadas. Fuente: elaboración propia

\begin{tabular}{|l|l|l|l|}
\hline \multicolumn{1}{|c|}{ TEMA } & \multicolumn{1}{|c|}{ CONTENIDO } & \multicolumn{1}{|c|}{$\begin{array}{c}\text { ACTIVIDAD } \\
\text { PRÁCTICA }\end{array}$} & \multicolumn{1}{c|}{ COMPETENCIAS } \\
\hline $\begin{array}{l}\text { 1. Conceptualización } \\
\text { de las relaciones } \\
\text { públicas }\end{array}$ & $\begin{array}{l}\text { Concepto y características. } \\
\text { Conceptualización en el } \\
\text { panorama científico: relación con } \\
\text { otras disciplinas. Funciones. } \\
\text { Problemas del término Sujetos y } \\
\text { estructuras. Proceso. }\end{array}$ & $\begin{array}{l}\text { Taller de oratoria. } \\
\text { Identificación de } \\
\text { materiales } \\
\text { comunicativos. } \\
\text { Anstrumentisis de textos y herramientas. } \\
\text { (sólo 1 } 1^{a} \text { ed.). } \\
\text { Proyecto grupal. } \\
\text { Ejercicio } \\
\text { individual. }\end{array}$ & $12,16,6,8,9,10,11$, \\
& & & \\
\hline
\end{tabular}

\begin{tabular}{|c|c|c|c|}
\hline $\begin{array}{l}\text { 2. Concepciones de las } \\
\text { relaciones públicas en } \\
\text { la historia }\end{array}$ & $\begin{array}{l}\text { Antecedentes y nacimiento. Era } \\
\text { de los pioneros. Mayoría de edad. } \\
\text { Cuatro modelos. Evolución en } \\
\text { España y Galicia. }\end{array}$ & Estudio de casos. & $1,2,10,11,12,15,16$ \\
\hline $\begin{array}{l}\text { 3. La profesión de las } \\
\text { relaciones públicas }\end{array}$ & $\begin{array}{l}\text { El profesional (formación, } \\
\text { características y cualidades). } \\
\text { Estructuras (departamentos y } \\
\text { consultoras). Asociaciones } \\
\text { profesionales. Límites } \\
\text { (legislación, ética y deontología } \\
\text { profesional). }\end{array}$ & $\begin{array}{l}\text { Ejercicio sobre } \\
\text { cuestiones éticas. } \\
\text { Debate sobre la } \\
\text { profesión (sólo } 1^{\text {a }} \\
\text { ed.) }\end{array}$ & $\begin{array}{l}4,5,7,9,10,11,13 \\
14,15,16\end{array}$ \\
\hline $\begin{array}{l}\text { 4. Públicos, opinión } \\
\text { pública y medios de } \\
\text { comunicación. }\end{array}$ & $\begin{array}{l}\text { Stakeholders, públicos y público } \\
\text { objetivo: conceptos, } \\
\text { clasificaciones y teorías. Concepto } \\
\text { y formación de la opinión } \\
\text { pública: la importancia de los } \\
\text { líderes de opinión. El papel de los } \\
\text { medios en la formación de la } \\
\text { opinión pública. }\end{array}$ & Estudio de casos. & $\begin{array}{l}4,8,10,11,12,15, \\
16\end{array}$ \\
\hline $\begin{array}{l}\text { 5. Relaciones públicas } \\
\text { aplicadas }\end{array}$ & $\begin{array}{l}\text { Relaciones públicas en la } \\
\text { empresa. Relaciones públicas, } \\
\text { política y gobierno. Relaciones } \\
\text { públicas en organizaciones } \\
\text { culturales, sociales y sanitarias. } \\
\text { Relaciones públicas en los } \\
\text { ámbitos del espectáculo, deportes } \\
\text { y turismo. }\end{array}$ & $\begin{array}{l}\text { Estudios de } \\
\text { casos. } \\
\text { Conferencia } \\
\text { profesional (sólo } \\
2^{\mathrm{a}} \text { ed.) }\end{array}$ & $8,9,10,11,12,15,16$ \\
\hline
\end{tabular}


programa de cinco temas que pretende dar cobertura a las competencias mencionadas en el epígrafe anterior y que busca, sobre todo, la familiarización del alumno con el lenguaje y los aspectos básicos de la disciplina, de tal modo que la materia sirva como punto de partida para los conocimientos que se deberán adquirir posteriormente en otras asignaturas. Además, cada tema teórico se relaciona con una o varias actividades prácticas, que contribuyen también a la adquisición de diversas competencias, especialmente aquellas relacionadas con el ser o saber estar.

El programa práctico, desarrollado en las sesiones de seminario y práctica, ha variado en algunos aspectos de la primera a la segunda edición de la asignatura, aunque ha mantenido su estructura esencial. Así, se ha optado por el desarrollo de diferentes actividades breves que el alumno puede realizar en el aula, con el asesoramiento del profesor y generalmente en grupo.

Teniendo en cuenta que a comienzo del curso el alumno carece de formación sobre relaciones públicas y que las sesiones prácticas comienzan a desarrollarse simultáneamente con las teóricas o muy poco después, para las primeras horas prácticas se propuso en ambas ediciones una actividad transversal que no exige conocimientos previos y que debe servir al alumno como herramienta para su actividad académica durante el desarrollo del grado y para su posterior futuro profesional. Se trata de un taller de oratoria en el que los alumnos reciben conocimientos básicos y eminentemente prácticos sobre comunicación verbal y no verbal, técnicas para hablar en público y mecanismos para realizar una presentación.

La segunda actividad práctica que se ha mantenido durante las dos ediciones de la materia ha sido el ejercicio de identificación de materiales comunicativos, que se realiza tras haber explicado las semejanzas y diferencias de varias disciplinas comunicativas en las sesiones teóricas. Esta práctica ayuda a los alumnos a clarificar los conceptos que han aprendido en la teoría, así como a observar que, aunque todas las disciplinas son teóricamente distintas e independientes, en la práctica es posible encontrar numerosos productos híbridos que, bien por sus objetivos o por su materialización, pueden ser asociados a diferentes formas comunicativas.

También se ha mantenido durante las dos ediciones la realización de un ejercicio sobre cuestiones éticas en el que se plantean diferentes casos o situaciones a las que se puede enfrentar el profesional de las relaciones públicas. Se pide a los alumnos que comprueben si los diferentes códigos deontológicos de uso habitual en la materia realizan alguna indicación al respecto y que se posicionen sobre la actuación que ellos llevarían a cabo. De este modo se familiarizan con los códigos y normas éticas de la profesión y valoran la dificultad de tomar decisiones en determinadas circunstancias. 
el aula diferentes estudiós de caso y ejerciciǒs de tipo práctico relacionados con diversos aspectos de interés abordados en las sesiones teóricas de la materia, como la comunicación de crisis, la generación de publicity para productos o el diseño de estrategias para campañas de comunicación pública. Muchos de los casos y ejercicios propuestos han sido seleccionados de los manuales de Wilcox, Cameron y Xifra (Wilcox, Cameron \& Xifra 2006) y Xifra y Lalueza (Xifra \& Lalueza, 2009).

El número de casos y ejercicios realizados en la segunda edición de la materia ha sido mayor que en la primera, ya que se han suprimido dos actividades cuyos resultados en la primera edición no fueron del todo satisfactorios: un ejercicio de análisis de textos con diferentes puntos de vista sobre la disciplina de las relaciones públicas, que no resultó adecuado porque implicaba un trabajo previo por parte de los alumnos que no se realizó; y un ejercicio de debate sobre la profesión de las relaciones públicas, sus límites y sus concomitancias con otras profesiones, que finalizó en un punto muerto por la falta de argumentos esgrimidos por los alumnos, como consecuencia de su todavía escasa formación en la materia y su dificultad, por tanto, para generar opiniones fundamentadas acerca de ciertos aspectos.

Por último, en la segunda edición se contempló como "actividad de aula" una conferencia impartida a final de curso por el director de comunicación de una empresa privada. Mediante esta actividad los alumnos se aproximaron de un modo más directo a la realidad de la profesión y comprobaron la aplicación de los diferentes conceptos teóricos estudiados al ámbito práctico.

Al margen de estas actividades, algunas de las sesiones prácticas se dedican en cada edición a la presentación, seguimiento y exposición de un proyecto grupal consistente, en ambas ediciones, en la creación de un dossier de presentación de una consultora de relaciones públicas y un plan de campaña elaborado por dicha consultora para la celebración del vigésimo aniversario de la Facultad.

Pese a la alta satisfacción del alumnado con este proyecto se detectaron dos problemas que aconsejan su reformulación de cara a futuras ediciones: un solapamiento con el tipo de trabajo que se propondrá para la asignatura Planificación estratégica de las relaciones públicas, que se imparte por primera vez en el curso 20112012 en $3^{\circ}$ de grado, y un aprovechamiento incompleto de las posibilidades del trabajo, teniendo en cuenta las limitaciones de los alumnos de $1^{\circ}$ de grado.

También se plantean en una de las sesiones prácticas las normas para la realización de un trabajo individual que, con el examen, permita al docente valorar el esfuerzo y evolución de cada alumno personalmente, al margen de su participación en las tareas de grupo. 
relaciones públicas en las organizaciones privadas. Se pedía al alumno que realizase una exposición lógica y coherente de argumentos basada en los conocimientos obtenidos en la materia y en las fuentes que considerase oportunas. Aunque algunos de los alumnos reflejaron en estos trabajos una comprensión importante de contenidos de la materia y una capacidad de argumentación aceptable, otros se limitaron a recopilar ideas de diversas fuentes (muchas de ellas procedentes de Internet e incorrectamente citadas) sin realizar una aportación propia verdaderamente relevante.

Teniendo en cuenta, por tanto, que este trabajo no resultó como esperaba la docente y habiendo detectado una carencia en los conocimientos de los alumnos sobre cómo citar adecuadamente las fuentes, en la segunda edición se optó por la realización de un trabajo encaminado a favorecer las habilidades investigadoras de los alumnos.

Para ello, se les pidió que localizasen diez definiciones de otros tantos términos estudiados en la materia, se les explicaron los procedimientos y fórmulas de citación y se establecieron diversas limitaciones para favorecer la investigación, relacionadas con el número de definiciones que podían proceder de la misma fuente, de Internet, o de referencias ofrecidas en la guía docente.

Este trabajo resultó muy útil para ayudar a los estudiantes a familiarizarse, de primera mano, con el modo correcto de realizar una cita y de referenciar una fuente, con resultados positivos a este respecto, pero no aportó información sobre la evolución de sus conocimientos personales sobre la materia.

\subsection{Metodologías aplicadas para la docencia}

\section{Sesiones teóricas}

Cada una de las sesiones docentes parte de una valoración de las ideas preconcebidas que muestran los alumnos en relación con diferentes conceptos de la materia. Desde esta perspectiva se procede a la exposición de los contenidos mediante el procedimiento de la lección magistral, apoyada con diferentes materiales impresos y audiovisuales que sirvan para ilustrar y ejemplificar tanto los conceptos teóricos como las diferentes técnicas y procesos explicados.

Tal y como apuntan Michavila y Zamorano, uno de los problemas de la clase magistral es que "puede fomentar la pasividad y la falta de participación del estudiante, lo que dificulta la reflexión sobre el aprendizaje y la falta de incentivos para la búsqueda de nuevos conocimientos" (Michavila y Zamorano, 2007). Por este motivo, durante estas presentaciones magistrales se intenta favorecer en la medida de lo posible, teniendo en cuenta el número de alumnos, la participación activa de los estudiantes, mediante preguntas y ejemplos que motiven los comentarios de los alumnos, que deben familiarizarse directamente con los conceptos que se manejan y con el uso de la bibliografía como fuente de conocimiento. 
En todas las prácticas se aplica una metodología de aprendizaje colaborativo en la que los alumnos trabajan en grupos, recogiendo en un documento escrito breve sus respuestas y justificaciones. A continuación, una vez finalizado el tiempo de análisis, los resultados se debaten de forma colectiva, lo que fomenta la participación de todos los alumnos y el intercambio de ideas y opiniones, a la vez que permite profundizar en las razones y cuestiones expuestas.

Tal y como hemos comentado en el apartado de contenidos, las metodologías específicas empleadas en estas sesiones prácticas y de seminario son muy diversas y han variado de la primera a la segunda edición en función del tipo de ejercicios desarrollados. En conjunto, en las dos ediciones se ha trabajado con las siguientes metodologías:

a) Seminario: trabajo en profundidad sobre los distintos temas, con ampliación y relación de los contenidos tratados en las sesiones magistrales (metodología aplicada en la primera edición a la actividad de análisis de textos).

b) Debate: actividad en la que dos grupos defienden posturas contrarias sobre un tema determinado (aplicada en la primera edición, con un debate sobre la profesión de las relaciones públicas).

c) Taller: actividades realizadas por los estudiantes con asistencia específica por parte del profesor, en este caso enfocadas a la adquisición de conocimientos y habilidades de comunicación verbal y no verbal.

d) Resolución de problemas y ejercicios: formulación, análisis, resolución y debate de un problema o ejercicio relacionado con la temática de la materia (aplicado a los ejercicios de identificación de materiales comunicativos o cuestiones éticas, entre otros).

e) Estudio de casos: análisis de un hecho, problema o caso real, con la finalidad de conocerlo, interpretarlo, resolverlo, generar hipótesis, contrastar datos, reflexionar, completar conocimientos, diagnosticarlo y adiestrarse en procedimientos alternativos de solución (aplicado, por ejemplo, al caso de comunicación de crisis).

f) Presentaciones y exposiciones: exposición oral por parte del alumnado de un tema concreto o un trabajo, previa presentación escrita (aplicada a los proyectos grupales).

\subsection{Sistema de evaluación}

Teniendo en cuenta la importancia de los conceptos teóricos por las características de la materia, se contempla la existencia de una prueba escrita aunque se apuesta por la diversidad de métodos de evaluación (véase Morán, 2008, p. 73). De este modo, se busca un sistema de valoración completo en el que se pueda cuantificar tanto la adquisición final de conocimientos y destrezas como el trabajo continuado del alumno o el trabajo en equipo. 
una puntuación mínima en la prueba escrita, según se especitica a continuación, y la obtención de una calificación global de al menos 5 puntos.

\section{El examen}

La adquisición de las competencias relacionadas con los contenidos teóricos se valora mediante la realización de una prueba tipo test cuya finalidad específica es medir el nivel de comprensión de los conceptos esenciales de la materia y el gado de profundidad de los conocimientos del alumno. La prueba se valora con un $40 \%$ de la puntuación final, es decir, con un máximo de cuatro puntos, y se exige al alumno que obtenga una puntuación mínima de 1,5 para que esta nota pueda ser sumada al resto de las calificaciones.

En la primera edición de la materia la prueba constó de un total de 40 preguntas cerradas con varias alternativas de respuesta de las que sólo una era correcta; cada respuesta correcta se valoraba con 0,1 puntos y cada respuesta errónea descontaba puntuación en la misma medida. En la segunda edición, con la finalidad de dar más valor a los conocimientos más importantes frente a otros menos esenciales, la prueba pasó a tener 35 preguntas, 10 de ellas valoradas con 0,15 puntos y las otras 25 valoradas con 0,1 puntos.

\section{Los trabajos entregados por los alumnos}

Tanto en la primera como en la segunda edición de la materia, los trabajos entregados por los alumnos supusieron el 35\% de la calificación final.

El proyecto grupal fue valorado con un máximo de 2,5 puntos teniendo en cuenta, teniendo en cuenta los siguientes aspectos: contenido, estructura, redacción, presentación y exposición. Por norma general, la calificación obtenida se aplicó de forma igualitaria a todos los integrantes de cada grupo.

En cuanto al trabajo individual se valoró en las dos ediciones con un máximo de 1 punto. En el trabajo de reflexión de la primera edición se valoró especialmente adecuación de los argumentos y su correcta exposición por escrito. En la segunda, se valoró la adecuación de las definiciones seleccionadas, la validez de las fuentes de acuerdo con los parámetros establecidos y la corrección a la hora de recoger las referencias.

\section{La observación cuantificada}

El 25\% restante de la nota corresponde a una observación cuantificada que realiza el docente sobre el trabajo regular y continuo del alumno en las prácticas de aula, teniendo en cuenta su participación activa en los talleres, seminarios y debates, así como la resolución de ejercicios, estudios de caso y las exposiciones que se realicen. 
sesiones, hasta el máximo total de 2,5 puntos. Cúando algún alumno justificó alguna falta de asistencia por razones válidas y documentadas, se le permitió realizar un breve trabajo compensatorio para recuperar la puntuación perdida en dicha sesión.

En la segunda edición se decidió flexibilizar este sistema, planteando para cada una de las sesiones una actividad alternativa de similar valor que todos los alumnos no asistentes, por causa justificada o no, podrían entregar en un plazo limitado (generalmente una semana después de la celebración de la práctica) para optar a recuperar la puntuación perdida.

\subsection{Resultados académicos}

Tabla 3. Porcentajes de alumnos por cada calificación en las dos ediciones de la materia. Fuente: Elaboración propia

\begin{tabular}{|c|c|c|c|c|c|c|c|c|c|c|}
\hline & \multicolumn{2}{|c|}{ AULA } & \multicolumn{2}{|c|}{ TRAB. INDIV. } & \multicolumn{2}{|c|}{ TRABAJO GRUPAL } & \multicolumn{2}{|c|}{ EXAMEN } & \multicolumn{2}{|c|}{ FINAL } \\
\hline ED & $1^{a}$ & $2^{\mathrm{a}}$ & $1^{\mathrm{a}}$ & $2^{\mathrm{a}}$ & $1^{a}$ & $2^{\mathrm{a}}$ & $1^{a}$ & $2^{\mathrm{a}}$ & $1^{\mathrm{a}}$ & $2^{\mathrm{a}}$ \\
\hline $\mathrm{MH}$ & 5,7 & 31,6 & 1,9 & 4,1 & 0 & 0 & 0 & 1,0 & 0 & 0 \\
\hline$S B$ & 24,8 & 32,7 & 14,3 & 30,6 & 5,7 & 12,2 & 4,8 & 9,2 & 4,7 & 15,3 \\
\hline $\mathrm{N}$ & 49,5 & 26,5 & 55,2 & 52,0 & 32,4 & 54,1 & 30,5 & 37,8 & 52,4 & 63,3 \\
\hline $\mathrm{AP}$ & 13,3 & 3,1 & 24,8 & 11,3 & 53,3 & 29,6 & 35,2 & 34,7 & 32,4 & 14,3 \\
\hline $\mathrm{SP}$ & 6,7 & 6,1 & 3,8 & 2,0 & 8,57 & 4,1 & 29,5 & 17,3 & 10,5 & 7,1 \\
\hline
\end{tabular}

En general, las calificaciones obtenidas por los alumnos en las dos ediciones de la materia fueron elevadas, como consecuencia, en gran parte, del sistema de evaluación, que permite ir acumulando puntos en diferentes apartados y que posibilita la obtención de una calificación de notable con relativa facilidad, aunque en la segunda edición de la materia se detecta un mayor porcentaje de alumnos con calificación final alta (notable y sobresaliente). Esta tendencia se reproduce en todas las calificaciones parciales de los alumnos.

Tanto en el caso del trabajo grupal, que no sufrió variaciones de planteamiento de la primera a la segunda edición, como en el caso del examen, donde los cambios introducidos no fueron especialmente relevantes a la hora de determinar resultados, en la segunda edición se incrementa el porcentaje de puntuaciones elevadas, mientras que desciende el porcentaje de alumnos que sólo habrían obtenido en el proyecto o en la prueba una nota equivalente al aprobado o al suspenso. 
se considerarían suspensos, que se reducen de forma muy signiticativa (de un $29,5 \%$ a un $17,3 \%)$.

También fueron mayores las calificaciones del trabajo individual de 2010/2011 que las del curso anterior, si bien en este hecho, además de la propia tendencia general del grupo, sí pudo influir la modificación del tipo de trabajo, que en la segunda edición resultó más mecánico y supuso una menor exigencia de reflexión por parte del alumno.

En el caso de la observación cuantificada, en la segunda edición se produjo un incremento muy considerable del porcentaje de alumnos que obtuvieron en este apartado la puntuación máxima (un 31,6\% frente a un 5,7\%), aspecto al que contribuyó, como era de esperar, el cambio en el procedimiento de puntuación y de recuperación de las sesiones perdidas expuesto anteriormente.

\subsection{Encuestas de evaluación docente}

Los alumnos valoran muy positivamente la preparación y organización de las clases teóricas, prácticas, resolución de ejercicios, etc., así como el ambiente docente (posibilidad de hacer preguntas, motivación para la participación, buen trato...), el nivel de aprendizaje conseguido y la adecuación de la metodología. Resulta muy positiva también la valoración general del docente y un poco menor, aunque también positiva, la valoración general de la materia, que mayoritariamente consideran importante para su formación, con contenidos interesantes, con un programa y bibliografía actualizados y con objetivos acordes a las horas lectivas. 


\begin{tabular}{|l|r|r|r|r|r|r|}
\cline { 2 - 8 } & \multicolumn{1}{|c|}{$\begin{array}{l}\text { PUNTUACIÓN } \\
\text { OBTENIDA }\end{array}$} & \multicolumn{4}{|c|}{$\begin{array}{c}\text { REFERENTES } \\
\text { (máx. 5) }\end{array}$} \\
\hline \hline & \multicolumn{2}{|c|}{ (máx. 5) } & \multicolumn{2}{c|}{ TITULACIÓN } & \multicolumn{2}{c|}{ UVIGO } \\
\cline { 2 - 8 } & $\mathbf{1}^{\mathrm{a}}$ ed. & $\mathbf{2}^{\mathrm{a}}$ ed. & $\mathbf{1}^{\mathrm{a}}$ ed. & $2^{\mathrm{a}}$ ed. & $\mathbf{1}^{\mathrm{a}}$ ed. & $2^{\mathrm{a}}$ ed. \\
\hline Organización de la materia & 4,52 & 4,54 & 3,59 & 3,67 & 3,7 & 3,69 \\
\hline Creación de un buen ambiente docente & 4,59 & 4,8 & 3,83 & 3,91 & 3,9 & 3,88 \\
\hline Nivel de aprendizaje conseguido & 4,5 & 4,32 & 3,49 & 3,56 & 3,58 & 3,57 \\
\hline Adecuación de la metodología & 4,41 & 4,36 & 3,38 & 3,43 & 3,48 & 3,46 \\
\hline Valoración general del docente & 4,73 & 4,6 & 3,77 & 3,7 & 3,78 & 3,71 \\
\hline Valoración general de la materia & 4,37 & 4,33 & 3,69 & 3,69 & 3,71 & 3,7 \\
\hline
\end{tabular}

Los resultados son parecidos en la primera y en la segunda edición de la materia, con variaciones poco significativas, y en todos los ítems la materia obtiene una valoración superior al referente de la Universidad de Vigo y de la titulación.

\section{6 trabajo del docente}

Como en todas las materias, esta asignatura implica para el docente un trabajo en cuatro frentes: tareas de preparación de la docencia y de materiales para los alumnos, horas lectivas, tareas de corrección y tareas de información y tutorización.

En cuanto al primer aspecto, en esta materia el docente prepara presentaciones de power point, selecciona textos de especial interés para su lectura por parte de los alumnos, busca o elabora los diferentes ejercicios que se desarrollan en las sesiones prácticas, crea los documentos explicativos para la elaboración de los trabajos grupales e individuales y planifica las actividades que se realizan fuera del aula. Además, se encarga de hacer disponibles los materiales a través de la plataforma de teledocencia.

En lo que se refiere a las horas lectivas, el reparto de los alumnos en 2 grupos para la docencia teórica y 4 grupos para la docencia de práctica y seminarios implica para el profesor una carga de 150 horas de docencia presencial en el aula.

Las tareas de corrección, por su parte, suponen una importantísima carga de trabajo para el docente. El profesor acumula hacia final de curso entre 110-120 exámenes tipo test, de corrección rápida; otros tantos trabajos individuales breves; y unos 20-25 trabajos grupales, cuya puntuación se realiza por un método comparativo, lo que obliga a revisar cada trabajo al menos en dos ocasiones. 
los alumnos van realizando para compensar sus ausencias en las sesiones prácticas y de seminario, lo cual supuso, con el sistema de la segunda edición, unos 10-15 ejercicios para corregir por cada práctica realizada en el aula, es decir, prácticamente cada semana. Cabe apuntar también que la modificación del trabajo individual en la segunda edición, al exigir una revisión muy minuciosa para comprobar la correcta citación de las fuentes, supuso, así mismo, un incremento importante en la carga del trabajo del profesor. Es necesario tener en cuenta, además, que por cada actividad o trabajo corregido el profesor emite un documento con las correcciones y comentarios que debe hacer llegar al alumno.

Por último, si bien la tutorización presencial no supone para el docente una inversión temporal importante, ya que las tutorías son voluntarias y los alumnos recurren a ellas en escasa medida, sí habría que computar el tiempo que el docente dedica a resolver las cuestiones que los alumnos plantean por correo electrónico o a enviar a través de la plataforma de teledocencia avisos sobre las actividades, plazos, feedback de las correcciones, calificaciones, etc.

\subsection{Modificaciones propuestas para la tercera edición}

A partir de los resultados del análisis detallado en los apartados anteriores, se considera que el desarrollo de la materia es positivo, en cuanto a aplicación de metodologías y resultados de evaluación, si bien conviene revisar algunos aspectos que solucionen ciertas deficiencias en la adquisición de competencias y que contribuyan a hacer más viable el trabajo del docente. De este modo, se propone mantener los contenidos del programa y la diversidad de metodologías aplicadas, aunque se reformula la propuesta de ejercicios y tareas y se revisa el sistema de evaluación.

Así, se propone, en primer lugar, modificar el sistema de calificación de las prácticas de aula, permitiendo la realización de trabajos compensatorios sólo ante la primera falta de asistencia no justificada o en todos los casos de ausencias justificadas. De este modo se espera reducir la carga de trabajo del profesor e incentivar la participación activa en las sesiones de trabajo práctico.

En segundo lugar, también con la finalidad de facilitar la labor de corrección del docente, se propone eliminar el trabajo individual e integrar las competencias que en él se trabajaban y la puntuación que se le concedía en el proyecto grupal y en el examen.

En tercer lugar, se propone sustituir el proyecto grupal por otro de carácter introductorio que prime la tarea de investigación. Incluirá en su primera parte una formulación teórica sobre las agencias de relaciones públicas, que deberá basarse en fuentes correctamente citadas (se cubre así el principal objetivo del trabajo individual planteado en la segunda edición). 
agencias de relaciones públicas nacional. Y la tercera parte consistirá én la elaboración de lo que en anteriores ediciones fue la primera parte del proyecto grupal, una presentación para una agencia de relaciones públicas de creación propia basada en los contenidos de los apartados anteriores. La puntuación del proyecto grupal se incrementará de un 25 a un 30\% de la puntuación final de la materia.

Por último, se mantendrá la formulación del examen tipo test, con un $40 \%$ de la puntuación final de la materia y el requisito mínimo de obtener un 1,5 sobre 4 puntos. Pero a este examen se le añadirá una pregunta breve de reflexión sobre una lectura obligatoria que se haya indicado durante el curso; esta pregunta se valorará con un máximo de medio punto $(5 \%)$ y sustituirá al trabajo individual de reflexión planteado en la primera edición de la materia.

\section{CONCLUSIONES}

En el presente artículo se han presentado las metodologías y sistema de evaluación aplicados en las dos primeras ediciones de una materia de grado del ámbito de las relaciones públicas, valorando su eficacia en términos de resultados académicos, de satisfacción del alumnado y de trabajo del docente.

En lo que se refiere a las metodologías docentes, se ha destacado la utilidad de contar con grupos reducidos en las sesiones prácticas, lo que permite aplicar un abanico de procedimientos metodológicos diversos y de carácter colaborativo para poder hacer frente a la materia desde diferentes ángulos y con fórmulas de trabajo distintas.

Esta pluralidad metodológica, que debe potenciarse en todo sistema docente en el espacio europeo de la educación superior, se complementa con un sistema de evaluación que, además de la prueba final y de los proyectos realizados de forma grupal o individual, valora la participación activa del alumno en las sesiones y su evolución en relación con las competencias que debe adquirir con la materia.

Tras dos ediciones de aplicación de este sistema, con algunas modificaciones de la primera a la segunda edición, se observa una alta satisfacción del alumnado y unos resultados académicos positivos, aunque a costa de una excesiva dedicación por parte del docente, sobre todo en términos de seguimiento y corrección de tareas.

Se propone, por tanto, para la tercera edición, la modificación de algunos elementos que permitan una racionalización del trabajo del docente y que a la vez resulten útiles para alcanzar un mayor aprovechamiento de los trabajos realizados y una mayor coordinación con otras materias de grado, manteniendo siempre las premisas básicas de pluralidad metodológica y de evaluación que se consideran esenciales para el correcto desarrollo de una materia de grado. 
De Miguel Díaz, M. (2006). Metodologías de enseñanza y aprendizaje para el desarrollo de competencias. Orientaciones para el profesorado universitario ante el Espacio Europeo de la Educación Superior. Madrid: Alianza Editorial.

Michavila, F. \& Zamorano, S. (2007). Reflexiones sobre los cambios metodológicos anunciados en la educación superior en España. Educación y futuro, 16: 31-46. Recuperado el 25 de septiembre de 2011, de http:/ / dialnet.unirioja.es/ servlet/articulo?codigo=2296442

Morán, P. (2008). El método de evaluación en el Espacio Europeo de la Educación Superior. Revista de Formación e Innovación Educativa Universitaria, 1(3): 7276.

Palomares Ruiz, A. (2011). El modelo docente universitario y el uso de nuevas metodologías en la enseñanza, aprendizaje y evaluación. Revista de Educación, 355: 591-604.

VV.AA. (1999). El espacio europeo de la enseñanza superior. Declaración conjunta de los ministros europeos de educación reunidos en Bolonia el 19 de junio de 1999. Recuperado el 20 de junio de 2011, de www.aneca.es/var/media/319432/rrii_declaracion_bolonia.pdf.

VV.AA. (2009). The Bologna Process 2020. The European Higher Education Area in the new decade. Communique of the Conference of European Ministers Responsible for Higher

Education. Recuperado el 20 de junio de 2011, de www.aneca.es/var/media/319456/rrii_declaracion_lovaina_eng.pdf

Wilcox, D. L., Cameron, G. T. \& XIFRA, J. (2006). Relaciones Públicas: estrategias y tácticas. Madrid: Pearson.

Xifra, J. \& Lalueza, F. (2009). Casos prácticos de relaciones públicas corporativas. Madrid: Pearson:

\section{María Isabel Míguez González}

Profesora contratada doctora en la Universidad de Vigo (España), donde imparte la materia de Teoría y práctica de las relaciones públicas, entre otras de este ámbito. Sus investigaciones se centran en temas de relaciones públicas, dirección de comunicación y turismo. Autora del libro Los públicos en las relaciones públicas. Ha publicado artículos en diversas revistas como Zer, Comunicación y sociedad, Razón y palabra, Revista Latina de Comunicación Social, Contratexto, Estudios de Periodismo y Relaciones Públicas $o$ Catalan Journal of Communication and Cultural Studies. 